\title{
General Theory of Body Contouring: 1. Processes of Quick and Slow Modulation of Subcutaneous Fat Tissue
}

\author{
Ilja L. Kruglikov \\ Wellcomet GmbH, Karlsruhe, Germany \\ Email: i.kruglikov@wellcomet.de
}

Received 25 February 2014; revised 20 March 2014; accepted 26 March 2014

Copyright (C) 2014 by author and Scientific Research Publishing Inc.

This work is licensed under the Creative Commons Attribution International License (CC BY).

http://creativecommons.org/licenses/by/4.0/

(c) (i) Open Access

\begin{abstract}
It is shown that modulation of subcutaneous white adipose tissue (sWAT) volume after application of different physical factors in body contouring procedures cannot be characterized by a single physiological reaction with dispersed reaction times, supposing that several different dynamic processes with different characteristic reaction times must be involved. Quick modulation of sWAT with characteristic times of $10^{3}-10^{5} \mathrm{~s}$ must be connected with such processes as hyaluronan production and consequent water retention in the tissue, as well as with reversible modulation of its mechanical properties. It is supposed that slow processes with characteristic times of up to $3 \times 10^{6} \mathrm{~s}$ can be caused by modulation of lipolytic activity of adipocytes through changes in their micro-environment or through their transdifferentiation, as well as through death and removal of adipocytes from sWAT.
\end{abstract}

\section{Keywords}

Body Contouring; Characteristic Reaction Time; Subcutaneous Fat Tissue; Volume Modulation; Hyaluronan

\section{Introduction}

Non-invasive and minimally invasive procedures actually belong to the main field of interest in aesthetic medicine. Classification of these procedures is normally determined according to the type of energy or energies they apply, e.g. ultrasound, infrared light, radio-frequency current, etc. Whereas application of every art of these energies demonstrate more or less pronounced (at least in some intervals) dose-effect dependence, very different distributions of absorbed energy in a given tissue of interest make it impossible to use the intensity of applied 
energy as a unique unit to compare the treatment results. This makes any objective comparison of different treatment methods difficult, leaving much room for speculation, especially concerning the physiological and biophysical pathways of their realization.

Subcutaneous white adipose tissue (sWAT) responds to different exogenous and endogenous factors with substantial modulation of its volume. This property is a core feature of sWAT, which is evidently or non-evidently used in different treatment methods in aesthetic medicine that are included in the group of so-called body contouring procedures. Moreover, while very different non-invasive methods in this field based on the application of various physical factors (e.g. constant and pulsed vacuums, RF currents, ultrasounds of different frequencies and intensities, heat and cold, light sources, etc.) demonstrate various dynamics of sWAT modulation, they often claim quantitatively comparable endpoint results, which clearly demonstrate that one or more nonspecific reactions must be involved in this effect.

Most theories proposed for different treatment methods of non-invasive body contouring assume at least one of two main mechanisms of sWAT modification-(apoptotic) cell death and/or (direct or indirect) stimulation of lipolysis - to be involved in observed sWAT volume modulation. Such an assumption seems to be necessary to ensure the prolonged effect of proposed treatment. At the same time, modulation of sWAT after such non-invasive, and some minimally invasive procedures can demonstrate very different characteristic reaction times needed to reach the endpoint, which can be the order of magnitude $\tau \sim 10^{3}-10^{7} \mathrm{~s}$. Additionally, such broad reaction time intervals point to the fact that not a single, but several, different physiological mechanisms must be involved in sWAT modulation and that these mechanisms are not necessarily connected with a simple cell death. Thus, the objective theory of body contouring must explain both the following phenomena: 1) the existence of a non-specific component or components in sWAT reaction and 2) the very broad intervals between reaction times. This can make the analysis of characteristic reaction times of sWAT modulation after the application of different physical procedures an important instrument to differentiate possible physiological pathways in observed endpoint results.

sWAT is a dynamic structure which has an average turnover rate of approximately $10 \%$ per year under normal conditions [1] [2], with a corresponding characteristic time of 10 years for full sWAT replacement. Assuming that this turnover rate is similar for different regional sWAT depots and that at least $10 \%$ of adipocytes in a given local sWAT depot must be replaced to see any significant local volume modulation, the characteristic time of the natural remodelling process in this tissue can be estimated as $\tau \sim 3 \times 10^{7} \mathrm{~s}$. Therefore, the observed volume modulation of sWAT after different body contouring procedures, which normally has much shorter characteristic reaction times, is not primarily connected with common processes of sWAT remodelling and must have other causes.

This raises the following questions: 1) How many and which physiological processes are responsible for a broad spectrum of reaction times in sWAT? 2) Which of these processes are responsible for a non-specific reaction of sWAT to different physical factors?

At the very least, a general theory of body contouring must take into account the following relevant properties of sWAT which have been formulated and investigated in recent years:

- Structural and physiological differences between hypertrophic and hyperplastic types of sWAT (see e.g. [1]

[2]), which naturally suppose the existence of distinct reactions of these types of sWAT to the same physical modalities [3];

- Special mechanical [4] [5] and viscoelastic [6] [7] properties of sWAT, which can be easily modified through the application of compression, shear stress and heating;

- Accumulation of hyaluronan (HA), and consequent of bounded water, in hypertrophic sWAT, demonstrating significant correlation with its degree of hypertrophy [8] [9], which can be responsible for the appearance of lipolytically resistant fat depots and play an important role in modulation of the thermo-mechanical properties of sWAT;

- Existence of local fibrosis (inter- or pericellular) in hypertrophic sWAT [10], which is evidently involved in the mechanical stability of sWAT [4] [5], can play an important role in the pathophysiology of cellulite [11] and also be modified through the application of different physical factors;

- Reversible processes of transdifferentiation between different types of adipocytes in sWAT (see e.g. [12]), as well as the production of "beige" adipocytes de novo [13], which can significantly modulate the volume and metabolic activity of sWAT.

These properties of sWAT are essential for the description of diverse effects observed in various procedures of body contouring as well as for making some non-obvious predictions in this field. Physiological pathways 
connected with these properties have different dynamic characteristics which can be sub-divided into quick and slow processes for classification purposes.

\section{Quick Dynamics of sWAT Volume Modulation}

Quick sWAT volume modification in response to different mechanical and thermic factors can be developed during several minutes up to one day (characteristic reaction times of $\tau \sim 10^{3}-10^{5} \mathrm{~s}$ ) and it must normally be connected with some non-specific reaction or reactions, since such modification can be observed by applying very different physical factors to the same local sWAT. This quick non-specific reaction cannot be caused by true fat tissue reduction through direct damage and destruction of adipocytes, through activation of delayed apoptotic cell death or through local activation of lipolysis in sWAT. All of these processes cannot be responsible for significant volume modulation, which is often observed immediately after a single treatment and can reach a value of over $1000 \mathrm{~cm}^{3}$.

\subsection{Modulation of Hyaluronan Content in sWAT}

As was proposed in [3] [14], this substantially non-specific quick reaction of sWAT could be connected with a local reduction of water content and is primarily dependent on significant regulation of hyaluronan (HA) content in a hypertrophic fat tissue. Such a mechanism evidently supposes different reactions of sWAT of different morphological structures, especially those of hypertrophic and normal or hyperplastic types, to the same physical factors.

HA has several remarkable physical properties, making its modulation important in body contouring. Among them are:

- High hydration and water retention capacities, which provide high osmotic pressure and allow quick regulation of tissue turgor, which correlates with modulation of sWAT volume;

- Very short turnover times, allowing quick regulation and adaptation of the thermo-mechanical properties of the tissue;

- High viscosity of HA solution which rapidly increases with HA concentration, $c$ (approximately as $c^{3.3}$ ) and can offer high resistance to a bulk water flow in sWAT;

- Pronounced shear dependent viscoelastic properties with low relaxation times, creating the possibility of a quick adaptation of HA containing system to a thermo-mechanical stress.

Because of the high HA turnover rate and its quick dynamic regulation, all these properties can be modified during very short times (minutes to hours). Additionally, HA turnover can be modulated through the application of different physical factors, which makes local adaptation of HA concentration (maybe, with fine tuning through modification of its molecular weight) an excellent candidate for a non-specific regulator with reactions observed during short characteristic times of $\tau \sim 10^{3}-10^{5} \mathrm{~s}$.

\subsection{Modulation of Mechanical Properties}

Although the majority of body contouring procedures declare the main goal of their application to be sWAT volume reduction, some of them claim to produce an increase in volume, which is supposed to be connected with the so-called "tightening" effect. Subcutaneous fat tissue permanently undergoes large deformations connected with the application of different mechanical forces and reacts to those with modification of its structure. From a mechanical point of view, sWAT can be described as a heterogeneous medium consisting of lipid-filled adipocytes embedded in an extracellular matrix. This matrix contains intercellular (septae) and pericellular (reinforcement membrane) collagen structures, which have different spatial distributions [4] [11].

The mechanical characteristics of sWAT are mainly connected with its pericellular collagen structure, which can be described as so-called closed-cell foam: single adipocytes form discrete pockets surrounded by their membranes reinforced by pericellular collagen. Contrarily, intercellular collagen structures form the coarsemeshed opened-cell foam (the internal pockets are connected with each other), which is known to have much worse mechanical characteristics as a closed-cell structure. Additionally, the elastic shear modulus of triglycerides in adipocytes is much lower than the total modulus of sWAT and can be also neglected if the mechanical properties of sWAT are considered [4]. The mechanical characteristics of this compound structure are strongly dependent on the relative density of the closed-cell structure, which is determined by adipocytes' size distribution and by the thickness of the pericellular fibrous structure around them. 
From the above, it is clear that a local re-distribution of cell sizes or reinforcement of pericellular structures can significantly shift the mechanical properties of sWAT. Mechanical properties of such a structure can be modulated by different physical factors, especially through the application of constant or cyclic mechanical stresses. Visible modulation of sWAT volume or of its stiffness demands, however, application of some critical number of stress-strain cycles, which corresponds to a critical local treatment time of one and the same body area. For example, the stiffness of sWAT can soar up to ten times after application of shear stress during $3 \times 10^{2}$ $-10^{3} \mathrm{~s}$ [7]. This treatment time is too long and will not normally be realized in dynamic body contouring methods were the applicators are moved over the body surface. This dramatic change of sWAT stiffness is reversible at least for relatively small strains and normally disappears with characteristic times of $\tau \sim 10^{4}$ s. On the contrary, the application of higher deformations can demonstrate the opposite but generally also the reversible effect-reduction of sWAT stiffness. Therefore, a quick mechanical modulation of sWAT volume is possible; however, it demands a prolonged local application of mechanical forces, which is almost never done in praxis, since it would dramatically increase the total treatment time of the applied procedure. Moreover, this sWAT modulation is reversible in almost all practical applications and will disappear during a very short time.

Another example of a "tightening” procedure is connected with the local application of an RF current. Collagen denaturation normally associated with its "shrinkage" was proposed to be the main effect of RF heating responsible for observed tissue tightening. However, the detailed analysis of this phenomenon leads to the conclusion that such an effect cannot be realised in vivo in an absolute majority of all treatment modalities [15] and that the observed shortcoming sWAT modulation can be connected with a local HA accumulation which is observed even during mild hyperthermia [16].

\section{Slow Dynamics of sWAT Volume Modulation}

Slow dynamic component of sWAT modification has a characteristic time of $1-4$ weeks $\left(\tau \sim 5 \times 10^{5}-3 \times 10^{6}\right.$ s) and can be connected with 1) modulation of lipolytic activity of adipocytes through a change in their micro-environment or through their transdifferentiation, 2) adipocytes' death and their removal from sWAT.

\subsection{Modification of Lipolytic Activity}

Local accumulation of HA in a vicinity of adipocytes is a hallmark of hypertrophic fat tissue [3] [8]. This accumulation was linked with different processes; among others, with those such as space filling, cell motility and adhesion, cell growth and differentiation. Significant accumulation of HA in a hypertrophic fat tissue was measured in animals fed with a high concentration of both glucose [8] and fat [9]. These studies demonstrated approximately a 3.5-fold and 2-fold increase of HA concentration in fat tissue, respectively. Such an increase of HA content results in significant accumulation of bounded water in hypertrophic sWAT and can lead to a reduction of AQP7 channels in adipocytes which are responsible both for water exchange and for glycerol efflux from these cells. A remarkable difference in AQP7 numbers in adipocytes was demonstrated between patients with normal BMI and in obesity [17] [18]. Such a deficiency of AQP7 in hypertrophic adipocytes can lead to sealing of adipocytes, making fat tissue more lipolytically resistant [3] and thus reducing its responsiveness to different stimulation procedures.

Although this HA accumulation is not microscopically spatially homogeneous and must be the highest in the vicinity of most hypertrophic adipocytes, this effect can tremendously modulate the total local metabolic activity of sWAT. To increase the lipolytic sensitivity of sWAT, its local HA content must first be significantly reduced, opening the limited timeframe for further metabolic stimulation of this tissue. Theoretically, the optimal treatment strategy based on these processes was described as TWL in [3]. It's most important feature is that any optimal treatment procedure must contain different treatment steps which should be provided one after another without overlapping, thus making the simultaneous application of different physical factors in one session less effective.

Concentration of HA in every tissue is balanced between the processes of its synthesis (through hyaluronan synthases, HAS) and degradation (through hyaluronidases, HYD). To reduce HA in sWAT for some sufficient time to cause its drainage and subsequent structural and/or metabolic changes, it would be necessary to either stimulate the production of HYD or suppress the activity of HAS, which is, for example, the case after application of different glucocorticoids (GC). If HA depletion in sWAT can really lead to a rapid sWAT volume reduction, then both GC and HYD application must demonstrate direct effect onto the fat mass (Figure 1). 


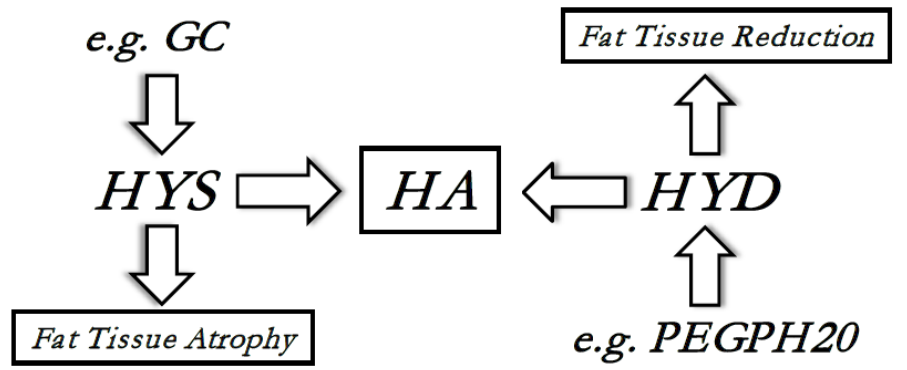

Figure 1. Dynamic balance of hyaluronan (HA): HA is produced by hyaluronan synthases (HYS) and is degraded by hyaluronidases (HYD). PEGPH20 is the pegylated form of hyaluronidase PH20 which can be used for prolonged intra-venous application of HYD and which effect onto the fat tissue was investigated in [9]; GC is a glucocorticoid, which can directly suppress the HYS activity [19] and can cause local tissue atrophy.

\subsection{Effect of Glucocorticoids on Lipolysis}

Application of GCs remains the first-line therapy in treatment of different inflammatory diseases in dermatology; it is also widely used in treatment of local pain. At the same time, this method demonstrates some severe adverse effects, among them skin atrophy. Another adverse effect, which can be often seen after subcutaneous injections of GCs, is local fat tissue atrophy.

Reports about the effects of GCs on adipose tissue are very contradictive, which is mainly connected with different physiological pathways involved in their metabolism [20]. Surprisingly, chronic GC exposure can stimulate both lipolysis and adipogenesis [21]. On the contrary, it is well known that direct GC treatment can cause elevated levels of circulating free fatty acids (FFAs) in plasma. This lipolytic activity of GC was long considered to be realized through genomic transcription. However, as was demonstrated in [19], GCs can directly stimulate lipolysis in adipocytes: application of dexamethasone caused lipolysis in a dose and time dependent manner and this stimulation could effectively be suppressed by direct application of GC receptor antagonists. It is significant that elevated FFA concentration in plasma, as well as glycerol release after dexamethasone treatment, correlated with increased lipase activity and with the intensity of lipolysis in vivo. Therefore, it can be concluded that the lipolytic pathway of GCs action is realized through the liberation of FFA efflux from adipocytes which was otherwise hindered.

Skin atrophy is considered to be a part of the natural aging process and it can be significantly enhanced by GC. It is well known that application of GCs over an extended period of time (normally several weeks) can lead to increased fragility, bruising and tearing of the skin. The side effects can be so severe that recently this condition received the special term "dermatoporosis" [22]. Additionally, it is known that GCs can reduce the proliferative activity of fibroblasts and decrease synthesis of collagen types I and III by these cells [23]. It can also significantly reduce the hyaluronan (HA) content in the skin [24]. Recently it was demonstrated that the rapid reduction of skin HA levels after GC application is connected with reduced synthesis of HA (through suppression of HAS-2) and that GCs do not directly influence the HA degradation process [25] [26]. Suppression of HA production, even after a short treatment period of just several days, can lead to a distinct reduction of HA levels in the skin and consequently to a substantial reduction of local water content in the treated areas.

Taken together, the application of GC (especially in a form of its subcutaneous injection) can rapidly deplete HA content in hypertrophic sWAT, especially in pericellular areas of adipocytes. This can improve the efflux of glycerol and liberation of FFAs from metabolically sealed adipocytes, which can effectively and locally reduce the sWAT volume.

\subsection{Effect of Hyaluronidases on Lipolysis}

Normally, the HYDs have very short half-life time, e.g. less as 3 min for PH20, which is the only HYD being active by neutral $\mathrm{pH}$ values. This restricts the experimental and clinical possibilities for long-term HA reduction. Recently, the experiments for depletion of HA content were done with a pegylated form of PH20, so called PEGPH20 [9], which has the serum half-life time of over 10 hours. 
To prove whether a long-term reduction of $\mathrm{HA}$ in fat tissue can really influence the deposition of triglycerides, the male C57BL/6 J mice were fed a chow or high fat (HF) diet (60\% of calories as fat) for 16 - 20 weeks [9]. It was demonstrated that the HF group developed hypertrophic fat tissue. Concentration of HA in the HF diet group was found to be 2-fold of its control value. Both control and HF groups were chronically intravenously treated (9 injections every 3 days) with PEGPH20 at $10 \mathrm{mg} / \mathrm{kg}$. The total time of this intervention was $\sim 3 \times 10^{6} \mathrm{~s}$, after which the content of HA in the tissue was almost completely depleted. As a result, the fat mass in these animals was totally decreased by approximately $35 \%$ and the significant reduction in the sizes of the adipocytes was revealed. It is significant that the observed fat reduction was not connected with the adipocytes' death, since the number of crown-like structures observed around the adipocytes was significantly reduced in the treatment against the vehicle group. Although the correlation between HA content, adipocyte size and the number of AQP7 in single adipocytes was not measured directly, these results demonstrate the crucial importance of HA accumulation in fat tissue, pointing to the significant dependence of metabolic activity of hypertrophic fat tissue on its HA content. This effect indirectly supports the theory of resistant fat depots described in [3], which connects the reduced lipolytic reaction in such depots with the hypertrophic nature of sWAT.

In this sense, both GCs and HYDs provide the "washout" phenomenon, effectively reducing the HA and consequently also the water content in the vicinity of hypertrophic adipocytes, thus producing a local "drainage" effect. This drainage effect was directly demonstrated in a combination of two body contouring methods [14]. At least for subjects with high BMI values there was a strong correlation between observed circumference reduction and total body water loss, which on average reached the value of $730 \mathrm{~mL}$ per $1 \mathrm{~cm}$ circumference reduction and which was registered at characteristic reaction times of $\tau \sim 3 \times 10^{3} \mathrm{~s}$.

Already this short analysis demonstrates significant differences in the homeostasis of hypertrophic and of normal/hyperplastic sWAT types. This must have a consequent impact on the reaction of these different types of sWAT to one and the same treatment procedure, making this phenomenon at least partly responsible for a wellknown high inter-subject variability of treatment results. Consequently, the development of optimal treatment procedures for modulation of sWAT may demand the differential diagnostics of sWAT types.

Since this is the principal item, we will summarize it once more, presenting different correlations which strongly support this picture (Table 1 ).

It would be very unusual if these multiple correlations existed only as artefacts. Moreover, they reflect the basic physiological processes and fundamental differences of hypertrophic versus normal or hyperplastic fat tissue. This questions the general assumption about homogeneity of the patients' cohort normally used in clinical studies into body contouring and demands much more critical evaluation of the common methods used for assessment of treatment results.

\subsection{Characteristic Times of Adipocytes' Death}

Not only a metabolic adaptation, but also the adipocyte death, can be directly responsible for slow dynamic component of sWAT modification. Indeed, dead adipocytes are removed from sWAT through macrophage phagocytosis, involving production of crown-like structures around the dead cells and lipid droplets [31]. The characteristic time for the removal of lipid droplets was determined, for example, in an inducible lipodystrophy model in the mouse, and it was estimated to be from several days up to 1 - 2 weeks [32] [33]. Additionally, one must take into account the eventual effect of delayed cell death which can have characteristic reaction times of 1 - 2 weeks. For example, it was reported that 15 minutes of thermal exposure of sWAT to $43^{\circ} \mathrm{C}-45^{\circ} \mathrm{C}$ can result in delayed adipocyte death of approximately 9 days [34]. Therefore, one can conclude that all significant modulations of sWAT volume reaching their end point during time interval of 1 - 4 weeks can theoretically involve the adipocyte death or eventually some significant delayed lipolytic reaction of adipocytes.

\subsection{Transdifferentiation-Qualitative Change of sWAT Structure}

Characteristic reaction time of up to $\tau \sim 3 \times 10^{6} \mathrm{~s}$ is also reasonable for the reaction of transdifferentiation (so called "britening") connected with the transformation of white adipocytes into a "beige" phenotype. Since "beige" cells are significantly smaller than the white adipocytes, such transdifferentiation can consequently reduce the sWAT volume [35]. It is important that such an effect can take place only if the total number of adipocytes remains almost constant which was also demonstrated in [35]. Production of "beige" cells in humans is connected with activation of so called UCP1 gene, normally through cold exposure or through $\beta_{3}$-adrenergic 
Table 1. Some correlations between the type of sWAT and its properties.

\begin{tabular}{|c|c|c|}
\hline Nr. & Correlation & Explanation \\
\hline 1 & $\begin{array}{l}\text { Spatial correlation between location of } \\
\text { hypertrophic adipocytes and degree of } \\
\text { lipolytic resistance of fat depots. }\end{array}$ & $\begin{array}{l}\text { Lipolytically more resistant sWAT in gluteo-femoral } \\
\text { depots consists of bigger adipocytes comparing to lipolytically } \\
\text { less resistant fat depots in other body areas [27]. }\end{array}$ \\
\hline 2 & $\begin{array}{l}\text { Spatial correlation between location of } \\
\text { hypertrophic adipocytes and HA } \\
\text { accumulation in fat tissue. }\end{array}$ & $\begin{array}{l}\text { There is a significantly higher accumulation of specific } \\
\text { HA in hypertrophic fat tissue compared to normal fat tissue [8] [9]. }\end{array}$ \\
\hline 3 & $\begin{array}{l}\text { Spatial correlation between HA content } \\
\text { and fibrosis in hypertrophic fat tissue. }\end{array}$ & Fibrosis is considered to be a hallmark of hypertrophic fat tissue [10]. \\
\hline 4 & $\begin{array}{l}\text { Spatial correlation between HA content and } \\
\text { induction of collagen types I and III in the tissue. }\end{array}$ & $\begin{array}{l}\text { This effect was clearly demonstrated e.g. by lung fibrosis, and it may } \\
\text { be of primary significance for hypertrophic and keloid scarring [28]. }\end{array}$ \\
\hline 5 & $\begin{array}{l}\text { Possible correlation between accumulation } \\
\text { of HA of some molecular weight with } \\
\text { production of collagen types IV and VI. }\end{array}$ & $\begin{array}{l}\text { Such correlation looks to be very reasonable but it had not been investigated } \\
\text { until now. If HAs really work as informational molecules [29], the molecular } \\
\text { weight of HA stimulating production of collagen types IV and VI has to be } \\
\text { different from that which induces fibrillar collagen types I and III. }\end{array}$ \\
\hline 6 & $\begin{array}{l}\text { Possible correlation between perivascular } \\
\text { fibrosis and disturbances of } \\
\text { micro-circulation in hypertrophic fat tissue. }\end{array}$ & $\begin{array}{l}\text { Although perivascular fibrosis in a hypertrophic fat tissue is well known [30] } \\
\text { and can lead to restriction of vasodilation, its spatial correlation with the } \\
\text { disturbance of micro-circulation in such tissue still has to be investigated. }\end{array}$ \\
\hline 7 & $\begin{array}{l}\text { Correlation between direct lipolytic effect } \\
\text { of glucocorticoids (GC) and their } \\
\text { ability to suppress the HA synthesis. }\end{array}$ & $\begin{array}{l}\text { GCs can quickly and effectively suppress the HA synthesis, which can } \\
\text { be the main reason for their atrophic action [26]. On the other hand, } \\
\text { GCs can directly stimulate lipolysis [19]. If these two effects } \\
\text { are connected to each other, the lipolytic effect of GCs can be } \\
\text { explained through removal of HA from adipocytes' surfaces, } \\
\text { which can temporally restore the lipolytic activity of these cells. }\end{array}$ \\
\hline 8 & $\begin{array}{l}\text { Correlation between local depletion of HA through } \\
\text { application of HYD and sWAT volume reduction. }\end{array}$ & $\begin{array}{l}\text { Chronic application of HYD leads to reduction of adipocytes' } \\
\text { volumes without excessive death of these cells [9]. }\end{array}$ \\
\hline
\end{tabular}

stimulation. This activation can take place in a timeframe of hours (up to $10^{4}-10^{5} \mathrm{~s}$ ). Without repeated treatments, this "britening" effect is reversible and disappears during a characteristic time of approximately 4 - 5 weeks ( 3 $\left.\times 10^{6} \mathrm{~s}\right)$ [12]. Similar characteristic times were also reported for the mechanism of "beige” adipocytes production de novo [13], which can be the main mechanism in at least some types of sWAT. However, it is uncertain as to whether such a mechanism would be important for the processes of body contouring. On the other hand, the same authors have demonstrated that, at least in some fat depots, a short cold application can surprisingly cause excessive white adipogenesis, i.e. fat hyperplasia. It is not clear what could be the reason for such a phenomenon; maybe the short cold application time gives no possibility to develop the full "beige" reaction as in chronic cold applications [36]. It is necessary to remember here that "surprising” fat hyperplasia was reported as a rare but severe complication after cold application in body contouring procedure named "cryolipolysis".

Repeated application of UCP1 stimulation procedure causes not only the "britening” of the same adipocytes but also the recruitment of new cells. Once produced, the beige adipocytes can retain in sWAT for some characteristic time. It was supposed that these cells are eliminated through normal sWAT turnover [36] with characteristic times of at least $\tau \sim 3 \times 10^{7}$ s. This can be the case e.g. in "cryolipolysis", where the first cold treatment would be needed to initiate the production of beige cells and the repeated treatments - to initiate the mechanism of thermogenesis in these cells.

Through mechanism of transdifferentiation, a heterogeneous fat tissue structure containing the mixture of clusters of small "beige" and of large white cells in the same sWAT can be produced. The reason for such clustering is not clear, but it evidently points to a limited spatial correlation radius of the involved process. Such a mixed structure has increased average lipolytic activity compared to pure sWAT, making such fat depots much more sensitive to forthcoming lipolytic stimulation. This forthcoming lipolytic stimulation must not be really done in a form of a special body contouring procedure, since it can be also caused by different pharmacological and nutritional agents through $\beta$-adrenergic stimulation [37]. It is almost obvious that in the case of slow dynamic modulation of sWAT the simultaneous application of different treatment modalities can theoretically increase the processes of transdifferentiation of adipocytes but cannot reduce the time to reach the endpoint of observed reaction. This treatment sequence is, after TWL as a treatment strategy for quick sWAT volume modula- 
tion [3] [38], another example of a temporal schedule in body contouring, taking into account a succession of different and generally non-overlapping treatment steps, where every previous step must be completed before the next one starts.

Production of spatial clusters containing adipocytes of different phenotypes can have another unexpected consequence for body contouring. These clusters can reach significant dimensions and sufficiently contribute to the development of non-homogeneous tension in fat tissue connected with its internal fibrosis. It is known that fibrosis (inter- and pericellular) is mainly developed in hypertrophic sWAT [10]. The appearance of fibrosis in the clusters of "beige" adipocytes must be very different from those in neighbouring clusters of hypertrophic white adipocytes, which can consequently produce additional tension on the boundary of such clusters. The consequence could be the skin appearance known as cellulite [11]. Interestingly, the yellow-brown colour which is typical for the "beige" adipocytes is often observed in body locations with cellulite [39].

\section{Conclusion}

Modulation of sWAT volume after application of different physical factors in body contouring procedures cannot be characterized by a single physiological reaction with dispersed reaction times. In contrast, several different dynamic processes with different characteristic reaction times must be involved. Quick modulation of sWAT with characteristic times of $10^{3}-10^{5}$ s must be connected with such processes as HA production and consequent water retention in the tissue. This modulation, by comparable amount of absorbed energy, is sufficiently independent on the type of applied physical factor, providing the highly non-specific and normally temporally transient component of sWAT reaction. Slow processes with characteristic times of up to $3 \times 10^{6} \mathrm{~s}$ can be caused by modulation of lipolytic activity of adipocytes through change of their type or of their micro-environment as well as through the death and removal of adipocytes from sWAT. Dynamic analysis gives no possibility of differentiating these reactions, meaning that different pathways can be responsible for similar endpoints in sWAT reaction.

\section{Conflict of Interests}

Dr. I. Kruglikov is the managing partner of Wellcomet $\mathrm{GmbH}$

\section{References}

[1] Spalding, K.L., Arner, E., Westermark, P. O., Bernard, S., Buchholz, B. A., Bergmann, O., Blomqvist, L., Hoffstedt, J., Näslund, E., Britton, T., Concha, H., Hassan, M., Ryden, M., Frisen, J. and Arner, P. (2008) Dynamics of Fat Cell Turnover in Humans. Nature, 453, 783-787. http://dx.doi.org/10.1038/nature06902

[2] Arner, E., Westermark, P., Spalding, K.L., Britton, T., Ryden, M., Frisen, J., Bernard, S. and Arner, P. (2010) Adipocyte Turnover: Relevance to Human Adipose Tissue Morphology. Diabetes, 59, 105-109. http://dx.doi.org/10.2337/db09-0942

[3] Kruglikov, I.L. (2012) Biophysical Basics of Body Treatments. Is Hyaluronan a Link That Has Gone Unnoticed? The American Journal of Cosmetic Surgery, 29, 121-129. http://dx.doi.org/10.5992/AJCS-D-12-00004.1

[4] Comley, K. and Fleck, N.A. (2010) A Micromechanical Model for the Young's Modulus of Adipose Tissue. International Journal of Solids and Structures, 47, 2982-2990. http://dx.doi.org/10.1016/j.ijsolstr.2010.07.001

[5] Comley, K. and Fleck, N.A. (2010) The Toughness of Adipose Tissue: Measurements and Physical Basis. Journal of Biomechanics, 43, 1823-1826. http://dx.doi.org/10.1016/j.jbiomech.2010.02.029

[6] Geerligs, M., Peters, G.W.M., Ackermans, P.A.J., Oomens, C.W.J. and Baaijens, F.P.T. (2008) Linear Viscoelastic Behavior of Subcutaneous Adipose Tissue. Biorheology, 45, 677-688.

[7] Geerligs, M., Peters, G.W.M., Ackermans, P.A.J., Oomens, C.W.J. and Baaijens, F.P.T. (2010) Does Subcutaneous Adipose Tissue Behave as an (Anti-)thixotropic Material? Journal of Biomechanics, 43, 1153-1159. http://dx.doi.org/10.1016/j.jbiomech.2009.11.037

[8] Han, C.Y., Subramanian, S., Chan, C.K., Omer, M., Chiba, T., Wight, T.N. and Chait, A. (2007) Adipocyte-Derived Serum Amyloid A3 and Hyaluronan Play a Role in Monocyte Recruitment and Adhesion. Diabetes, 56, 2260-2273. http://dx.doi.org/10.2337/db07-0218

[9] Kang, L., Lantier, L., Kennedy, A.J., Bonner, J.S., Mayes, W.H., Bracy, D.P., Bookbinder, L.H., Hasty, A.H., Thompson, C.B. and Wasserman, D.H. (2013) Hyaluronan Accumulates with High Fat Feeding and Contributes to Insulin Resistance. Diabetes, 62, 1888-1896. http://dx.doi.org/10.2337/db12-1502 
[10] Khan, T., Muise, E.S., Iyengar, P., Wang, Z.V., Chandalia, M., Abate, N., Zhang, B.B., Bonaldo, P., Chua, S. and Scherer, P.E. (2009) Metabolic Dysregulation and Adipose Tissue Fibrosis: Role of Collagen VI. Molecular and Cellular Biology, 29, 1575-1591. http://dx.doi.org/10.1128/MCB.01300-08

[11] Kruglikov, I.L. (2012) The Pathophysiology of Cellulite: Can the Puzzle Eventually Be Solved? Journal of Cosmetics, Dermatological Sciences and Applications, 2, 1-7. http://dx.doi.org/10.4236/jcdsa.2012.21001

[12] Rosenwald, M., Perdikari, A., Rülicke, T. and Wolfrum, C. (2013) Bi-Directional Interconversion of Brite and White Adipocytes. Nature Cell Biology, 15, 659-667. http://dx.doi.org/10.1038/ncb2740

[13] Wang, Q.A., Tao, C., Gupta, R.K. and Scherer, P.E. (2013) Tracking AdipogenesisDuring White Adipose Tissue Development, Expansion and Regeneration. Nature Cell Biology, 19, 1338-1344. http://dx.doi.org/10.1038/nm.3324

[14] Kruglikov, I.L. (2012) Biophysical Basics of Body Treatments: A Preliminary Study into the Correlation between Washout and Circumference Reduction. The American Journal of Cosmetic Surgery, 29, 196-202.

[15] Kruglikov, I.L. (2013) Neocollagenesis in Non-Invasive Aesthetic Treatments. Journal of Cosmetics, Dermatological Sciences and Applications, 3, 1-5. http://dx.doi.org/10.4236/jcdsa.2013.31A001

[16] Wegrowski, Y. (1993) Effect of Hyperthermia on the Extracellular Matrix. I. Heat Enhances Hyaluronan and Inhibits Sulphated Glycosaminoglycan Synthesis. FEBS Letters, 334, 121-124. http://dx.doi.org/10.1016/0014-5793(93)81695-V

[17] Ceperuelo-Mallafre, V., Miranda, M., Chacon, M.R., Vilarrasa, N., Megia, A., Gutierrez, C., Fernandez-Real, J.M., Gomez, J.M., Caubet, E., Frühbeck, G. and Vendrell, J. (2007) Adipose Tissue Expression of the Glycerol Channel Aquaporin-7 Gene Is Altered in Severe Obesity but Not in Type 2 Diabetes. Journal of Clinical Endocrinology \& Metabolism, 92, 3640-3645. http://dx.doi.org/10.1210/jc.2007-0531

[18] Prudente, S., Flex, E., Morini, E., Turchi, F., Capponi, D., De Cosmo, S., Tassi, V., Guida, V., Avogaro, A., Folli, F., Maiami, F., Frittitta, L., Dallapiccola, B. and Trischitta, V. (2007) A Functional Variant of the Adipocyte Glycerol Channel Aquaporin 7 Gene Is Associated With Obesity and Related Metabolic Abnormalities. Diabetes, 56, 1468-1474. http://dx.doi.org/10.2337/db06-1389

[19] Xu, C., He, J., Jiang, H., Zu, L., Zhai, W., Pu, S. and G. Xu, (2009) Direct Effect of Glucocorticoids on Lipolysis in Adipocytes. Molecular Endocrinology, 23, 1161-1170. http://dx.doi.org/10.1210/me.2008-0464

[20] Peckett, A.J., Wright, D.C. and Riddell, M.C. (2011) The Effects of Glucocorticoids on Adipose Tissue Lipid Metabolism. Metabolism Clinical and Experimental, 60, 1500-1510. http://dx.doi.org/10.1016/j.metabol.2011.06.012

[21] Campbell, J.E., Peckett, A.J., D’souza, A.M., Hawke, T.J. and Riddell, M.C. (2011) Adipogenic and Lipolytic Effects of Chronic Glucocorticoid Exposure. American Journal of Physiology-Cell Physiology, 300, C198-C209. http://dx.doi.org/10.1152/ajpcell.00045.2010

[22] Kaya, G. and Saurat, J.H. (2007) Dermatoporosis: A Chronic Cutaneous Insufficiency/Fragility Syndrome. Clinicopathological Features, Mechanisms, Preventation and Potential Treatments. Dermatology, 215, 284-294. http://dx.doi.org/10.1159/000107621

[23] Schoepe, S., Schacke, H., May, E. and Asadullah, K. (2006) Glucocorticoid Therapy-Induced Skin Atrophy. Experimental Dermatology, 15, 406-420. http://dx.doi.org/10.1111/j.0906-6705.2006.00435.X

[24] Kaya, G., Tran, C., Sorg, O., Hotz, R., Grand, D., Carraux, P., Didierjean, L., Stamenkovic, I. and Saurat, J.-H. (2006) Hyaluronate Fragments Reserve Skin Atrophy by CD44-Dependent Mechanism. PLoS Medicine, 3, e493. http://dx.doi.org/10.1371/journal.pmed.0030493

[25] Zhang, W., Watson, C.E., Liu, C., Williams, K.J. and Werth, V.P. (2000) Glucocorticoids Induce a Near-Total Suppression of Hyaluronan Synthase mRNA in Dermal Fibroblasts and in Osteoblasts: A Molecular Mechanism Contributing to Organ Atrophy. Biochemical Journal, 349, 91-97. http://dx.doi.org/10.1042/0264-6021:3490091

[26] Gebhardt, C., Averbeck, M., Diedenhofen, N., Willenberg, A., Anderegg, U., Sleeman, J.P. and Simon, J. (2010) Dermal Hyaluronan Is Rapidly Reduced by Topical Treatment with Glucocorticoids. Journal of Investigative Dermatology, 130, 141-149. http://dx.doi.org/10.1038/jid.2009.210

[27] Tchoukalova, Y.D., Koutsari, C., Karpyak, M.V., Votruba, S.B., Wendland, E. and Jensen, M.D. (2008) Subcutaneous Adipocytes Size and Body Fat Distribution. American Journal of Clinical Nutrition, 87, 56-63.

[28] Wilkinson, T.S., Potter-Perigo, S., Tsoi, C., Altman, L.C. and Wight, T.N. (2004) Pro- and Anti-Inflammatory Factors Cooperate to Control Hyaluronan Synthesis in Lung Fibroblasts. American Journal of Respiratory Cell and Molecular Biology, 31, 92-99. http://dx.doi.org/10.1165/rcmb.2003-03800C

[29] Stern, R., Asari, A.A. and Sugahara, K.N. (2006) Hyaluronan Fragments: An Information-Rich System. European Journal of Cell Biology, 85, 699-715. http://dx.doi.org/10.1016/j.ejcb.2006.05.009

[30] Divoux, A., Tordjman, J., Lacasa, D., Veyrie, N., Hugol, D., Aissat, A., Basdevant, A., Guerre-Millo, M., Poitou, C., Zucker, J.-D., Bedossa, P. and Clement, K. (2010) Fibrosis in Human Adipose Tissue: Composition, Distribution, and 
Link with Lipid Metabolism and Fat Mass Loss. Diabetes, 59, 2817-2825. http://dx.doi.org/10.2337/db10-0585

[31] Cinti, S., Mitchell, G., Barbatelli, G., Murano, I., Ceresi, E., Faloia, E., Wang, S., Fortier, M., Greenberg, A.S. and Obin, M.S. (2005) Adipocyte Death Defines Macrophage Localization and Function in Adipose Tissue to Obese Mice and Humans. Journal of Lipid Research, 46, 2347-2355. http://dx.doi.org/10.1194/jlr.M500294-JLR200

[32] Pajvani, U.B., Trujillo, M.E., Combs, T.P., Iyengar, P., Jelicks, L., Roth, K.A., Kitsis, R.N. and Scherer, P.E. (2005) Fat Apoptosis through Targeted Activation of Caspase 8: A New Mouse Model of Inducible and Reversible Lipoatrophy. Nature Medicine, 11, 797-803. http://dx.doi.org/10.1038/nm1262

[33] Sun, K., Kusminski, C.M. and Scherer, P.E. (2011) Adipose Tissue Remodelling and Obesity. The Journal of Clinical Investigations, 121, 2094-2101. http://dx.doi.org/10.1172/JCI45887

[34] Franco, W., Kothare, A., Ronan, S.J., Grekin, R.C. and McCalmont, T.H. (2010) Hyperthermic Injury to Adipocyte Cells by Selective Heating of Subcutaneous Fat with a Novel Radiofrequency Device: Feasibility Studies. Lasers in Surgery and Medicine, 42, 361-370. http://dx.doi.org/10.1002/lsm.20925

[35] Vitali, A., Murano, I., Zingaretti, M.C., Frontini, A., Ricquier, D. and Cinti, S. (2012) The Adipose Organ of Obesity-Prone C57BL/6J Mice Is Composed of Mixed White and Brown Adipocytes. Journal of Lipid Research, 53, 619629. http://dx.doi.org/10.1194/jlr.M018846

[36] Harms, M. and Seale, P. (2013) Brown and Beige Fat: Development, Function and Therapeutic Potential. Nature Medicine, 19, 1252-1263. http://dx.doi.org/10.1038/nm.3361

[37] Bonet, M.L., Oliver, P. and Palou, A. (2012) Pharmacological and Nutritional Agents Promoting Browning of White Adipose Tissue. Biochimica et Biophysica Acta (BBA)-Molecular and Cell Biology of Lipids, 1831, 969-985. http://dx.doi.org/10.1016/j.bbalip.2012.12.002

[38] Kruglikov, I.L. (2013) Is the Depletion of Hyaluronan in Hypertrophic Fat Tissue a Key Event in Body-Contouring Procedures? The American Journal of Cosmetic Surgery, 30, 244-245.

[39] Kruglikov, I.L. (2013) Kontroversen in der Ästhetischen Medizin. 7. Kryolipolyse-Apoptose vs. Thermogenese. Kosmetische Medizin, 34, 202-207.

\section{Abbrevations}

AQP-aquaglyceroporin,

BMI—body mass index,

FFAs - free fatty acids,

GC-glycocorticoids,

HA-hyaluronan,

HAS-hyaluronan synthases,

HYD—hyaluronidase,

sWAT - subcutaneous white adipose tissue,

PEGPH20 - pegylated form of PH20,

$\mathrm{UCP}$ - uncoupling protein. 